\title{
MRPPSim: A Multi-Robot Path Planning Simulation
}

\author{
Ebtehal Turki Saho Alotaibi, Hisham Al-Rawi \\ Computer Science Department, Al Imam Muhammad Ibn Saud Islamic University \\ Riyadh, SA
}

\begin{abstract}
Multi-robot path planning problem is an interesting problem of research having great potential for several optimization problems in the world. In multi-robot path planning problem domain (MRPP), robots must move from their start locations to their goal locations avoiding collisions with each other. MRPP is a relevant problem in several domains, including; automatic packages inside a warehouse, automated guided vehicles, planetary exploration, robotics mining, and video games. This work introduces MRPPSim; a new modeling, evaluation and simulation tool for multi-robot path planning algorithms and its applications. In doing so, it handles all the aspects related to the multi-robot path planning algorithms. Through its working, MRPPSim unifies the representation for the input. This algorithm provides researchers with a set of evaluation models with each of them serving a set of objectives. It provides a comprehensive method to evaluate and compare the algorithm's performance to the ones that solve public benchmark problems inas shown in literature. The work presented in this paper also provides a complete tool to reformat and control user input, critical small benchmark, biconnected, random and grid problems. Once all of this is performed, it calculates the common performance measurements of multi-robot path planning algorithms in a unified way. The work presented in this paper animates the results so the researchers can follow their algorithms' executions. In addition, MRPPSim is designed as set of models, each is dedicated to a specific function, this allows new algorithm, evaluation model, or performance measurements to be easily plugged into the simulator.
\end{abstract}

Keywords-component; simulation; modeling; evaluation; multi-robot path planning problem; performance measurements

\section{INTRODUCTION}

Formally, Multi-robot path planning problem (MRPP) consists of a graph and a set of robots. In such problems, each robot has to reach its destination in the minimum time with minimum number of moves. MPP is a relevant problem in a wide range of domains, including; automatic packages inside a warehouse [2], automated guided vehicles [3], planetary exploration [4], robotics mining [5], and video games [6].

There are several variants of MRPP algorithms in the literature with their specialized strengths. However, to have clear vision about these algorithms' performance, these need to be evaluated on a unified robust tool. The multi-robot path planning simulation (MRPPSim) is a modeling, evaluation and simulation tool for multi-robot path planning algorithms and its applications. It handles all the aspects related to the multi-robot path planning algorithms. Hence, the researchers need only to worry about their algorithms. MRPPSim aims to provide the researcher set of evaluation models, each of them serves one of the following objectives;
Objective.1: Test and track the algorithm's behavior for specific cases in fully controlled problems.

Objective.2: Track the algorithm's behavior and compare its performance with the algorithms that are already evaluated on predefined small critical problems in [7].

Objective.3: Test the algorithm's behavior on very large graphs when the occupying ratio (robots number/vertices count) increases.

Objective.4: Compare the algorithm's performance to the performance of the algorithms already tested in publically available large scale problems [1] such as [7-9].

Objective.5: Compare the algorithm's performance to the performance of the algorithms solves biconnected graphs [10], $[7,8]$

Objective.6: Test the algorithm's behavior on fixed biconnected graph's size when the occupying ratio increases.

Objective.7: Evaluate the algorithm's performance against the algorithms that solve random graphs.

Objective.8: Track the algorithm's behavior on fixed random graph's size when the occupying ratio increases.

Objective.9: Compare the algorithm's performance to the algorithms that solve grid graphs [7, 8, 10].

Objective.10: Test the algorithm behavior on fixed grid graph's size when the occupying ratio increases.

Objective.11: Evaluate the algorithm's performance on biconnected graphs of fixed occupying ratio when the graph's size increases.

Objective.12: Evaluate the algorithm's performance on random graphs of fixed occupying ratio when the graph's size increases.

Objective.13: Evaluate the algorithm's performance on grid graphs of fixed occupying ratio when the graph's size increases.

This paper is organized as follows; after this introduction, the problem is defined in Section II. In Section III, the multirobot path planning simulation MRPPSim is introduced. In Section IV, MRPPSim objectives are discussed in details. Finally, conclusions and future works are presented in Section V.

\section{PROBLEM STATEMENT}

Going through the literature related to MRPP algorithm, we found an urgent need of having a graph-based multi-robot path 
planning simulator that can cover different types of scenarios and problems. The study of all available algorithms implementations and its performance measurements calculations as well was essential. The simulation is to provide a ready implementation of state-of-art algorithms in the field. During this study, the work started by looking into iRRT simulator [11]. iRRT is a simple Java program for simulating that is widely used for robotics path planning algorithm known as Rapidly-exploring Random Tree or RRT. This algorithm was developed by Sertac Karaman et al. [12] and it works for a single-robot configuration. During the process, several extensions were implemented to fit multi-robot RRT requirements. However, the representation of the robots environment was limited to being continuous environment, while we were also interested in graph-based algorithms. This would enable working with problem scenarios and variations that have not been catered for previously.

\section{METHODOLOGY}

The proposed work, i.e. MRPPSim is written as an open source $\mathrm{C}++$ code $^{1}$ designed to be fully modularized so that researchers can have the ability to plug any modifications.

\section{A. Input}

The input parameter for MRPPSim can be classified based on the simulation model which will be described in the next section. In our implementation, the user is initially required to select the simulation model and the algorithm to be used. Based on that, the user can enter or select the main two input for any algorithm. These inputs include; the representation of the environment in the form of undirected graph; $G(V, E)$, whatever the simulation model or the algorithm, the graph that would be sent to the algorithm in XML format representation describing numbered vertices $V$, and the linking edges $E$ (Fig.1). The second input is the instance; $I(R$, Locations $)$, which is the representation of the number of robots; $R$, and robots' configuration within the environment; Locations, the robots configuration is the definition of the robots start and goal locations in graph's vertices term. (Fig.2).

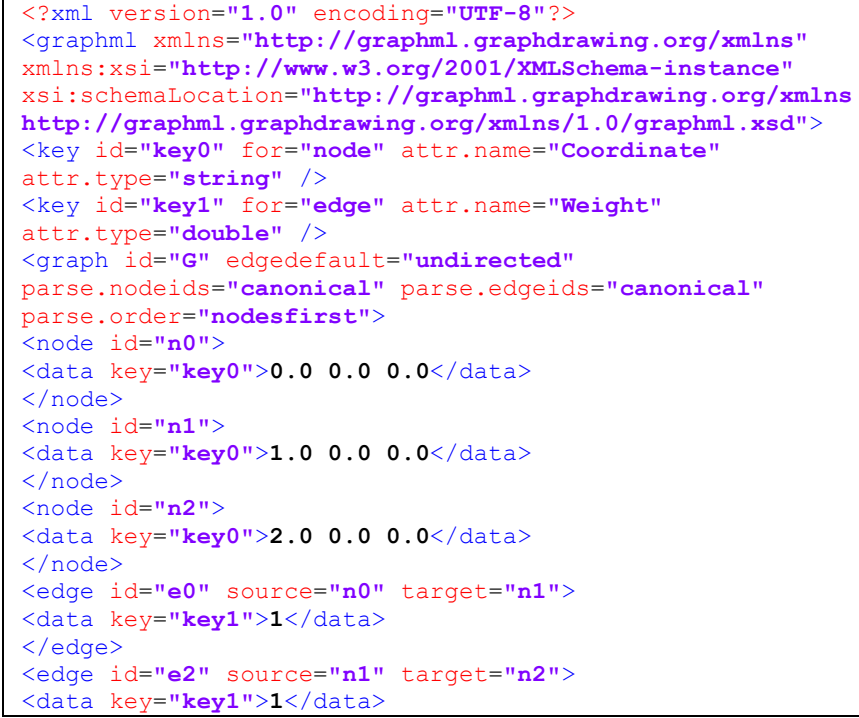

\footnotetext{
${ }^{1}$ https://pushandspin.wordpress.com/source-code/
}

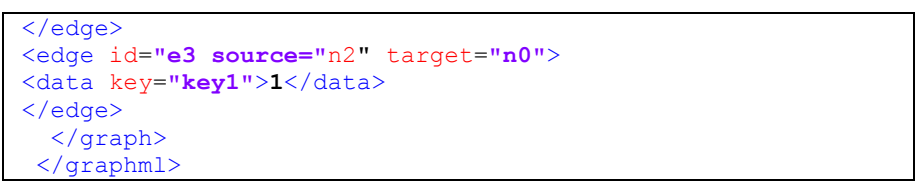

Fig. 1. The environment representation is a graph in $\mathrm{xml}$ format

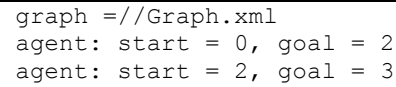

Fig. 2. The robots (agents) start and goal locations (instance) representation is a text file in specific format

\section{B. Simulation models}

As already mentioned, MRPPSim is written as a $\mathrm{C}++$ code that offers a set of simulation models, Each of these can serve as set of objectives in our problem definition. This section will present an overview of each simulation model in detail starting with the user input until the algorithm inputs which is the graph and the instance, described earlier, in the following form;

\section{1) Fully controlled model}

\section{AlgorithmX ( $G, I)$}

In this selection, the user will be able to set the attributes of both graph and the instance to be tested. Once the user enters the graph $G$ (user defined, user defined) attributes and the instance $I$ (user defined) descriptions, MRPPSim then converts the input to the form defined in the subsection $A$, and send these inputs to the selected algorithm as;

\section{AlgorithmX (G(user defined), I (user defined)).}

The aim of this model is to provide the user with the ability to track specific cases in fully controlled problem (Objective.1).

\section{2) Small benchmark model}

In order to cater for a wider class of instances, MRPPSim has defined six benchmark problems evaluated in [7], a tree with cycle leaves problem evaluated in [11] and a biconnected graph in [10](Fig.3). It is noteworthy that besides the graph $G$ (ready $V$, ready $E$ ), the instance is also defined in these problems as $I$ (ready $R$, ready locations). Therefore, the algorithm input would be;

AlgorithmX $(\mathrm{G}$ (ready $V$, ready $E), \quad I($ ready $R$, ready locations)).

The aim of this model is to get the user with the ability to compare the algorithm with already evaluated algorithm on these problems (Objective.2).

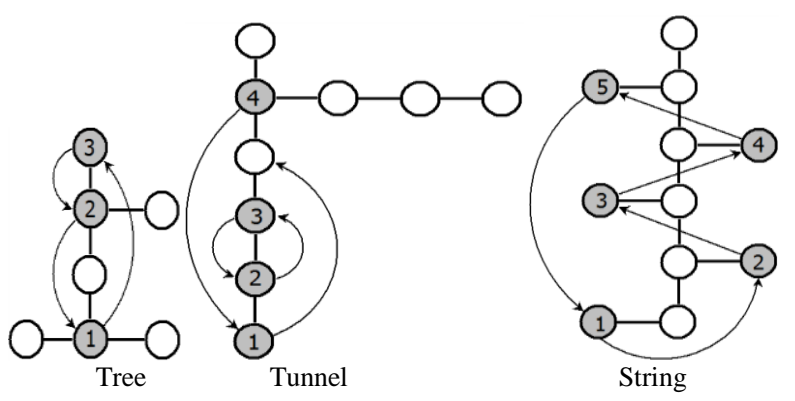




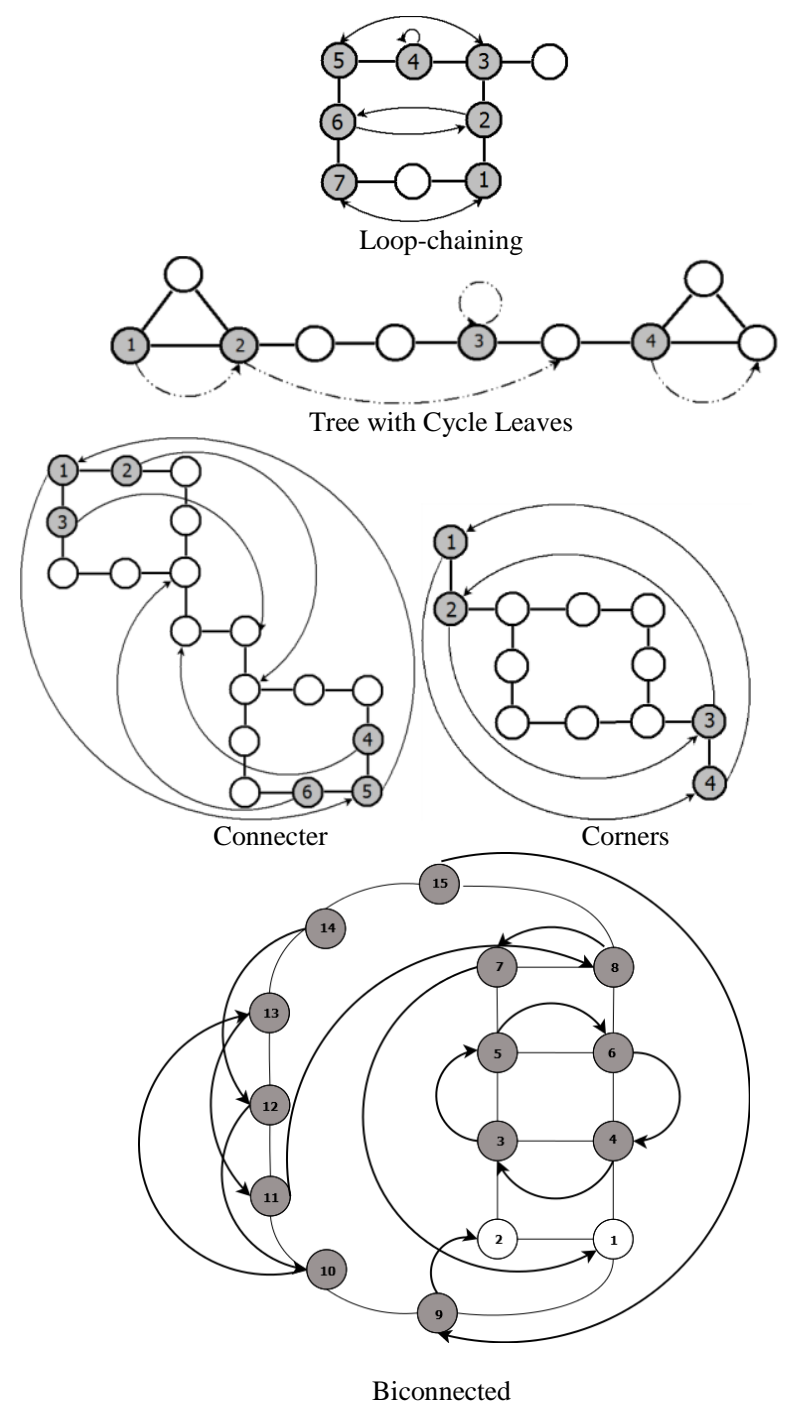

Fig. 3. Small benchmark problems

\section{3) Public benchmark model}

Sturtevant in his paper [1] has presented a new repository that has been placed online ${ }^{2}$ to improve the evaluation of gridbased problems. The repository allows researchers to use the same problems and test sets, thereby increasing the reproducibility of published results. In the repository, two main sets are described; Commercial Game Benchmarks and Artificial Benchmarks. Each map has been coded in a way to be parsed. The maps are attached with a set of scenarios describing the instances. However, this repository requires a little modification in order to be applicable to the chosen problem domain. This is due to the fact that in some scenarios, there would be multiple robots on the same start and/or goal position. Consequently, instead of using attached scenarios, we have designed random generator. It is used to generate any number of robots for the given graph randomly. The procedures for generating random instances is;

1. Parameter: $n$ : the number of vertices, $r$ : the number of robots.

\footnotetext{
${ }^{2}$ http://movingai.com/benchmarks/
}

2. For each $r$;

1. Select random node $s$ such that $s \notin$ start set

2. Set $s$ as starting vertex of $r$.

3. $\quad$ start set $=$ start set $\mathrm{U} s$

4. Select random vertex $g$ such that $s \notin$ goal set

5. Set $s$ as goal vertex of $r$.

6. goal set $=$ goal set $\cup$ g.

In the Commercial Game Benchmarks, the maps contain areas of land or water and the robots can only move on water. (Fig.4,5). Moreover, in the Artificial Benchmarks, the maps contain areas of passageways and walls. We have designed a parser to read the coded maps and transform them into graphs with each element in the map converted to vertex if it not obstacles (lands/walls). This results in graphs that are characterized by a large set of connected vertices. The parser generates a fixed graph representation; $G(\operatorname{coded} V$, coded $E$ ), for every coded map. In addition set of instances describing the start and goal locations would also be generated for each graph randomly by the generator given the number of robots $R$, minimum number of robots, maximum number of robots and the step size; $I\left(R, \sum_{i=0}^{\max } \min +(i *\right.$ StepSize $)$, random locations). Hence, The algorithms input would be;

\section{AlgorithmX ( $G(\operatorname{coded} V$, coded $E)$,}

$I\left(R, \sum_{i=0}^{\max } \min +(i *\right.$ StepSize $)$,random locations $\left.)\right)$.

The aim of this model is to test the algorithm behavior on very large graphs when the occupying ratio (robots number/vertices count) increases (Objective.3) and to compare the algorithm's performance to the performance of the algorithms already tested in these public problems (Objective.4).

\section{4) One-Factor controlled model}

While studying Multi-Robot Path Planning problem, we came across two critical factors affecting the algorithms' performance. These are the size of the graph and the occupying ratio (number of robots/ number of vertices). In this section we will overview two type of models; each type controlling one of these critical factor and randomize the other in order to generate graphs and instance files.

\section{a) Fixed-Graph with Variable-Robot model}

In this model, number of instances are to be generated for the same graph size. After setting the graph size, the user can set the minimum number of robots, maximum number of robots and the step size to generate required number of instances varying in the occupying ratio.

\section{BICONNECTED GRAPH}

The biconnected graph is a connected graph on two or more vertices having no articulation vertices. MRPPSim provides Biconnected graph generator representing $G\left(V, E_{b i}\right)$ with the number of vertices; $V$. The generator will generate the biconnected topology by inserting edges $E_{b i}$ between these vertices. In this version of MRPPSim we have used the same biconnected graph generator in [10]. It generates random biconnected graphs conforming to three parameters; $h$ the number of handles, $C_{0}$ the size of the initial cycle, and $l$ the maximum handle length (Fig.6). 


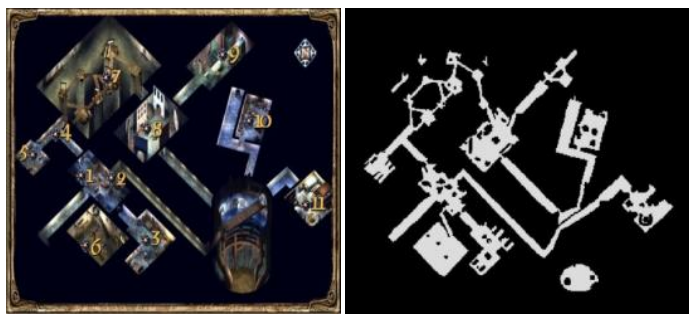

Fig. 4. AR0306SR map of Baldurs Gate II (left), its coded map (right)
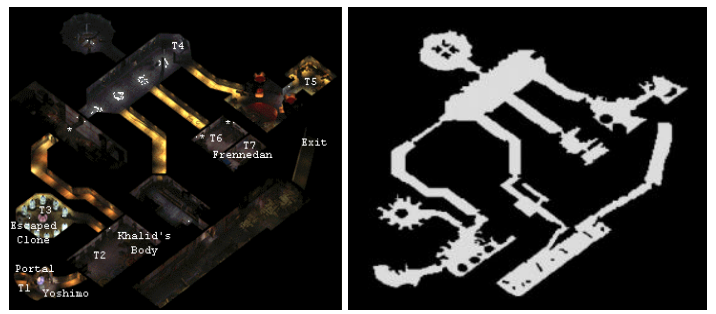

Fig. 5. AR0603SR map of Baldurs Gate II (left), its coded map (right)

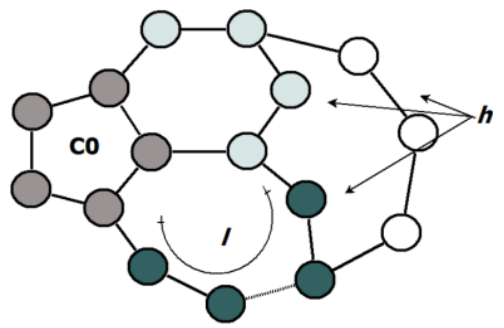

Fig. 6. initial cycle (C0), maximum handle length (1) and number off handles (h) in Surynek grap

Since this model is FG-VR model, the instances $I$ need to be generated randomly. The user can set the minimum number of robots, maximum number of robots and the step size to generate a number of instances varying in the occupying ratio; $\sum_{i=0}^{\max } \min +(i * S t e p S i z e)$. However, the robots location; locations need to be randomized totally. Furthermore, The algorithm input would be;

$\operatorname{AlgorithmX~}\left(G\left(h, C_{0}, l\right)\right.$,

$I\left(\sum_{i=0}^{\max } \min +(i *\right.$ StepSize $)$, random locations $\left.)\right)$.

The aim of this model is to provide the researcher with the ability to compare the algorithm's performance to the performance of the algorithms thereby meeting objective 5 and 6 mentioned in earlier sections.

\section{RANDOM GRAPH}

MRPPSim provides random graph generator; $G(V, E)$ given the number of vertices; $V$, the generator would generate the random topology by inserting edges $E_{\text {rand }}$ between these vertices. Since this model is FG-VR model, the instances $I$ will be generated randomly. As has been described earlier, the user can set the minimum number of robots, maximum number of robots and the step size to generate number instances varying in the occupying ratio; $\sum_{i=0}^{\max } \min +(i *$ StepSize $)$, the robots location; locations will be randomized totally. Therefore, the algorithm input would be;

\footnotetext{
$\operatorname{AlgorithmX}\left(G\left(V, E_{\text {rand }}\right)\right.$,
}

$I\left(\sum_{i=0}^{\max } \min +(i *\right.$ StepSize $)$, random locations $\left.)\right)$

The aim of this model is to provide the researcher with the ability to evaluate the algorithm performance against the performance of algorithms already exusting that solve random graphs (Objective.7) and to track algorithm behavior on fixed random graph's size when the occupying ratio increases. (Objective.8).

\section{GRID GRAPH}

MRPPSim provides random graph generator; $G(V, E)$ given the number of vertices; $V$, the generator would generate the random topology by inserting edges $E_{\text {grid }}$ between these vertices. In this version of MRPPSim, We have used the same generator in [10] to generate grid instances of $n \times n$ size with a constant value of the parameter $\left(h, C_{0}, l\right)=G\left((n-1)^{2}-1,4,4\right)$. Since this model is FG-VR model, the instances $I$ would be generated randomly. On the other hand, the user can set the minimum number of robots, maximum number of robots and the step size to generate a number of instances varying in the occupying ratio; $\sum_{i=0}^{\max } \min +(i *$ StepSize $)$, the robots location; locations will be randomized totally. The algorithm input thus would be;

AlgorithmX $\left(G\left((n-1)^{2}-1,4,4\right)\right.$,

$I\left(\sum_{i=0}^{\max } \min +(i *\right.$ StepSize $)$, random locations $\left.)\right)$.

The aim of this model is to get the researcher with the ability to compare the algorithm's performance to the performance of the algorithms that solve grid graphs, [8], [7]. (Objective.9) and to evaluate the grid graphs when the occupying ratio increase (Objective.10).

\section{b) Fixed-Robot with Variable-Graph model}

In this model, a number of graphs will be generated for the same occupying ratio (number of robots/number of vertices). After setting the robot number, the user can set the minimum number of vertices, maximum number of vertices and the step size to generate a number of graphs varying in their size.

\section{BICONNECTED GRAPH}

Since we have used the same biconnected graph generator as presented in [10], the graph would be generated according to a single variable $x$ that can range from the given minimum number of vertices to the given maximum number of vertices by the given step size. The variable $x$ would be used for all three parameters (number of handles; $h$, initial cycle size; $C_{0}$, and maximum handle length; $l$ ). The graph size $V$ hence would be $C_{0}+h * l$. The number of empty vertices would be kept fixed to the given occupying ratio. Hence, the instance would be $I$ (occupying ratio * $V$, random location). The algorithm input will be:

AlgorithmX $\left(G(x, x, x), I\left(\right.\right.$ occupying ratio $*\left(x+x^{*} x\right)$, random location) ),

such that, $x=\sum_{i=0}^{\max } \min +(i *$ StepSize $)$.

The aim of this model is to get the researcher with the ability to evaluate algorithm's performance on biconnected graphs of fixed occupying ratio when the graph's size increases. (Objective.11).

\section{RANDOM GRAPH}


After the researcher has set the occupying ratio, minimum number of vertices, maximum number of vertices and the step size, the random generator would automatically generate graph $G\left(V_{i}=\sum_{i=0}^{\max } \min +(i *\right.$ StepSize $\left.), E_{\text {rand }}\right)$. The instance would be $I$ ( $V_{i}$ * occupying ratio, random locations ). Hence, the algorithm input would be:

$$
\operatorname{AlgorithmX}\left(\mathrm{G}\left(\mathrm{V}_{\mathrm{i}}=\sum_{i=0}^{\max } \min +(i * \text { StepSize }), \mathrm{E}_{\mathrm{rand}}\right),\right.
$$

$\mathrm{I}\left(\mathrm{V}_{\mathrm{i}} *\right.$ occupying ratio, random locations $\left.)\right)$.

The aim of this model is to get the researcher with the ability to evaluate the algorithm performance on random graphs of fixed occupying ratio when the graph's size increases. (Objective.12).

\section{GRID GRAPH}

After the researcher has set the occupying ratio, the minimum number of vertices, maximum number of vertices and the step size. MRPPSim would automatically generate different grids $\left.G\left(V_{i}=\sum_{i=0}^{\max } \min +(\mathrm{i}-1)^{2}-1,4,4\right), E_{\text {grid }}\right)$ with the same occupying ratio. The instances would be $I\left(V_{i}^{*}\right.$ occupying ratio, random locations). Therefore, the algorithm input would be;

AlgorithmX $\left(\mathrm{G}\left(\sum_{i=0}^{\max } \min +(\mathrm{i}-1)^{2}-1,4,4\right), \mathrm{E}_{\text {grid }}\right)$, $\mathrm{I}\left(\mathrm{V}_{\mathrm{i}}{ }^{*}\right.$ occupying ratio, random locations $\left.)\right)$.

The aim of this model is to evaluate the algorithm performance on grid graphs of fixed occupying ratio when the graph's size increases (Objective.13).

\section{Output}

The most common performance measurements of multirobot path planning algorithms in $[7,8,10]$ are;

1) The path length

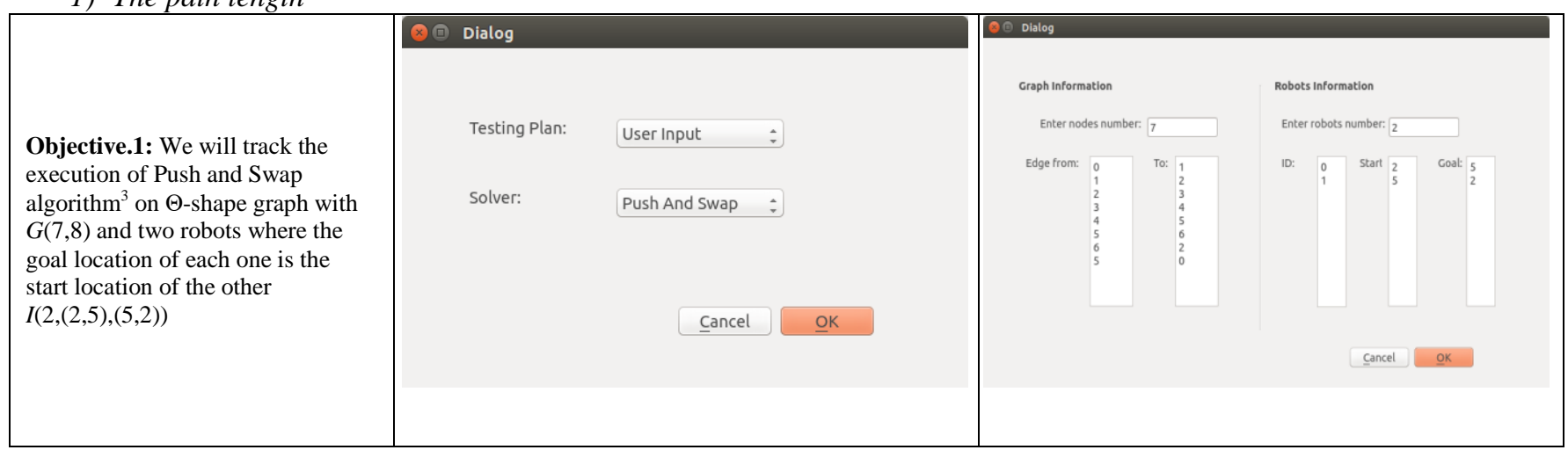

Even though some algorithms in literature have implemented parallel implementation, our contribution in this work is to unify the performance parameters allowing the researchers to calculate the path length as the total number of sequential moves.

\section{2) The CPU time}

The execution time has been calculated as the real time between the algorithm start time to the end time. In addition, we have ignored the execution time of the preprocess of the algorithms.

\section{3) The makespan}

Makespan is the number of time steps required to get all robots to their destination.

4) The optimized path length

These measurements carry any improvement of path length on the original algorithm.

\section{5) The optimized makespan}

These measurements carry any improvement of makespan on the original algorithm.

The animation results would be written in the format readable to GraphRec simulator [14]. On its completion, all these results would be stored in the experiment folder. The researchers can then track the movement and the execution of the algorithm by running the animation results in GraphRec.

\section{RESULTS}

The results of this work are aimed to verify whether the objectives described earlier are satisfied or not. In this section, we will recall every objective and show the ability of MRPPSim to achieve it. 


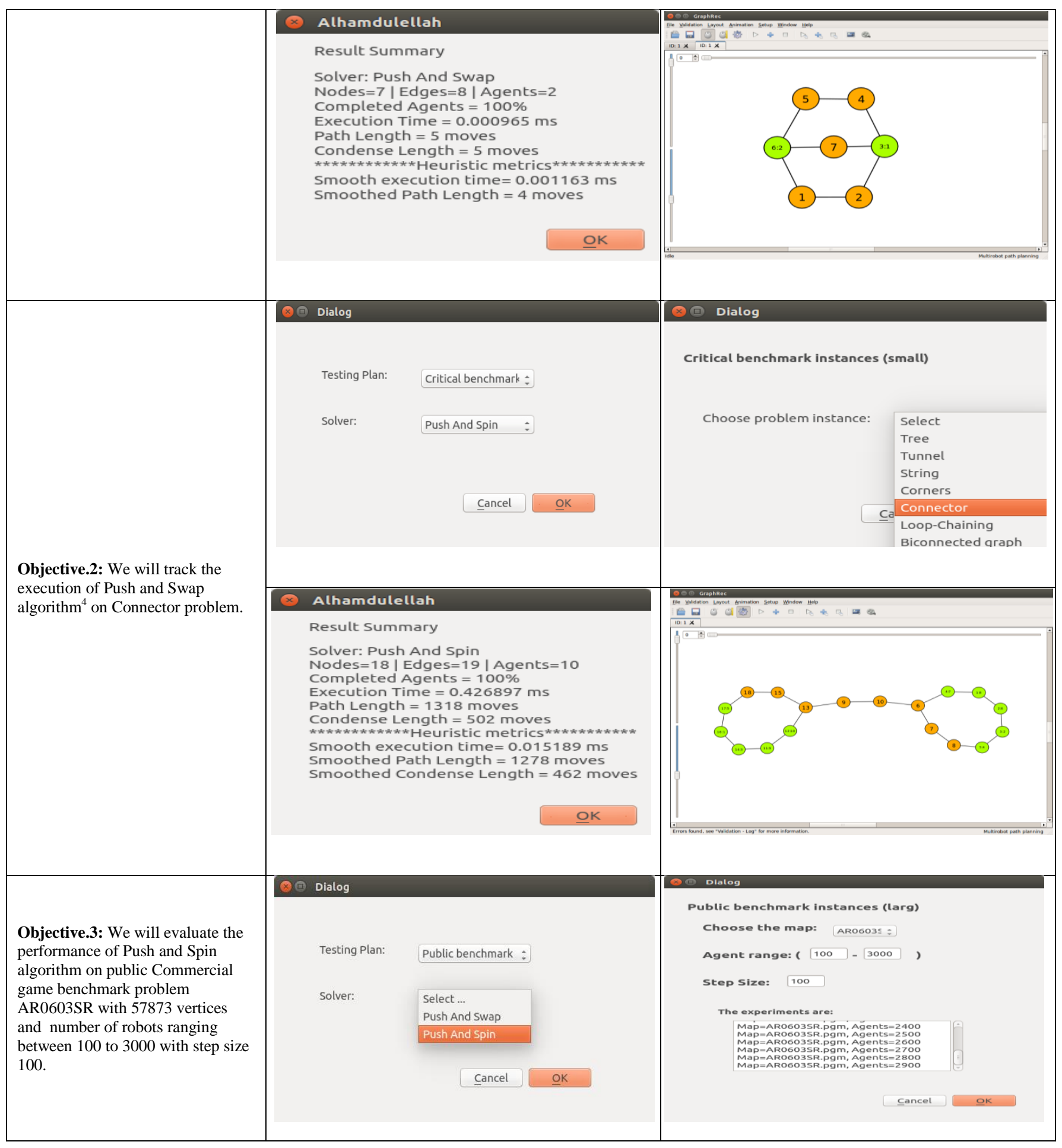

\footnotetext{
${ }^{4}$ One of the implemented algorithm in MRPPSim
} 


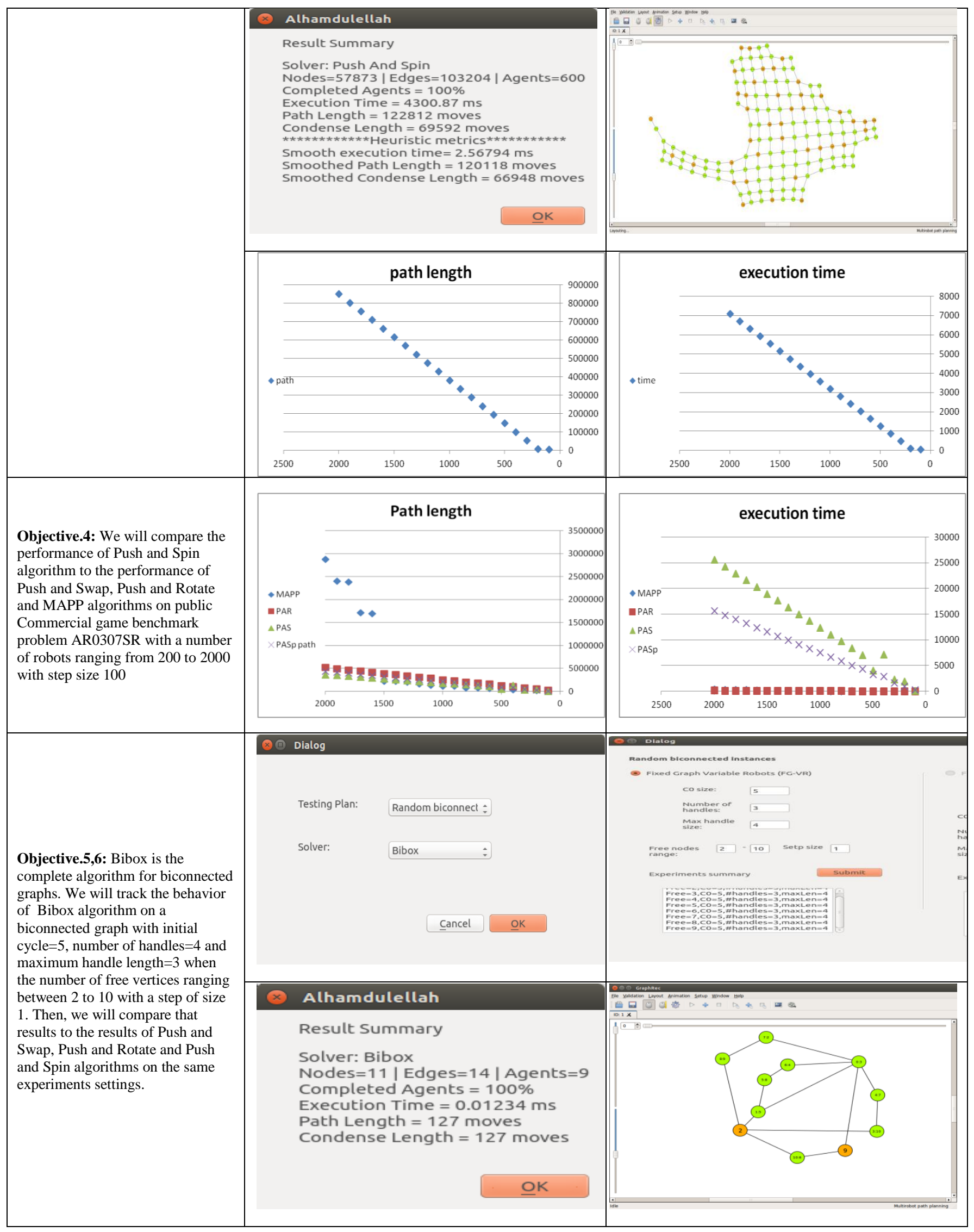




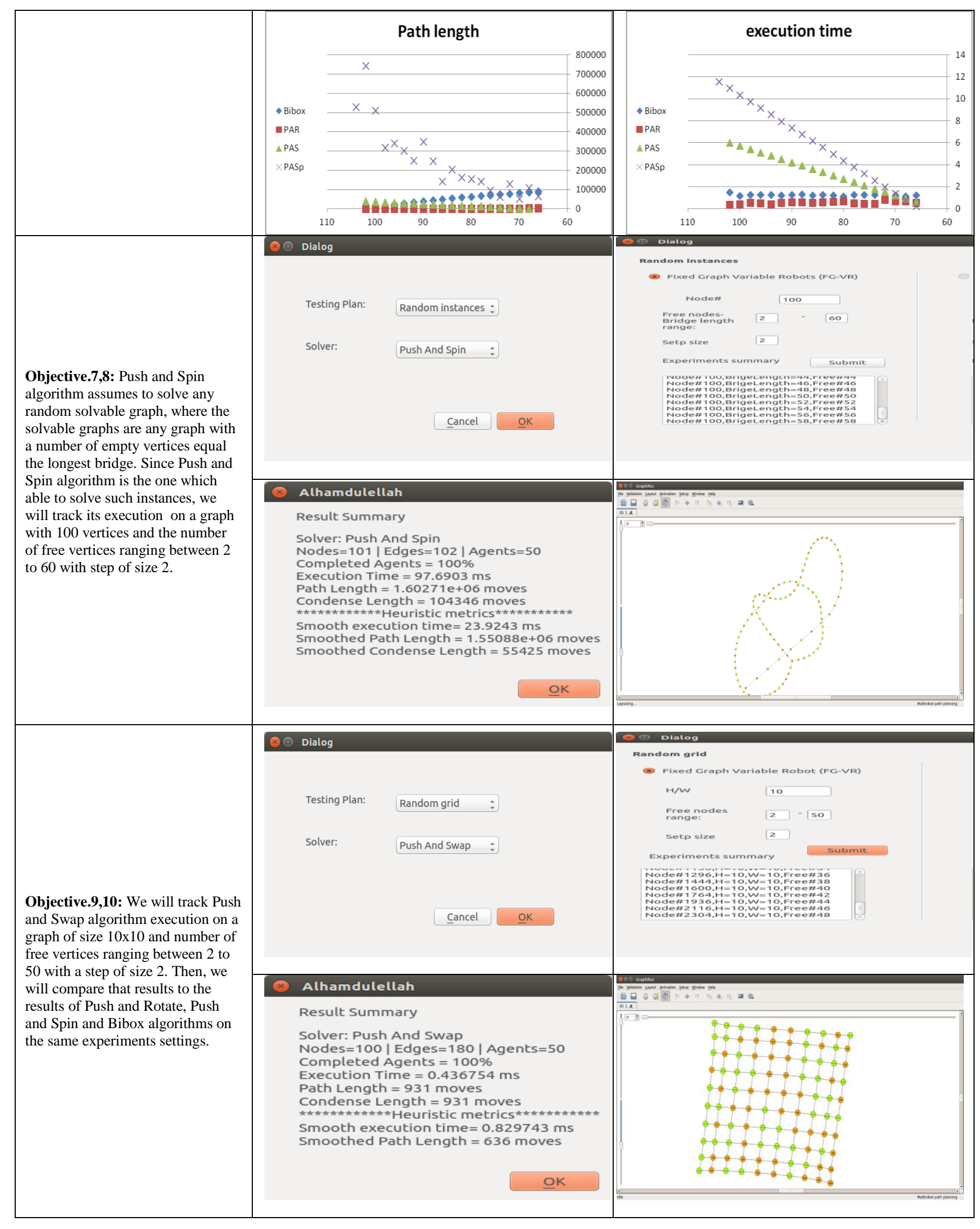




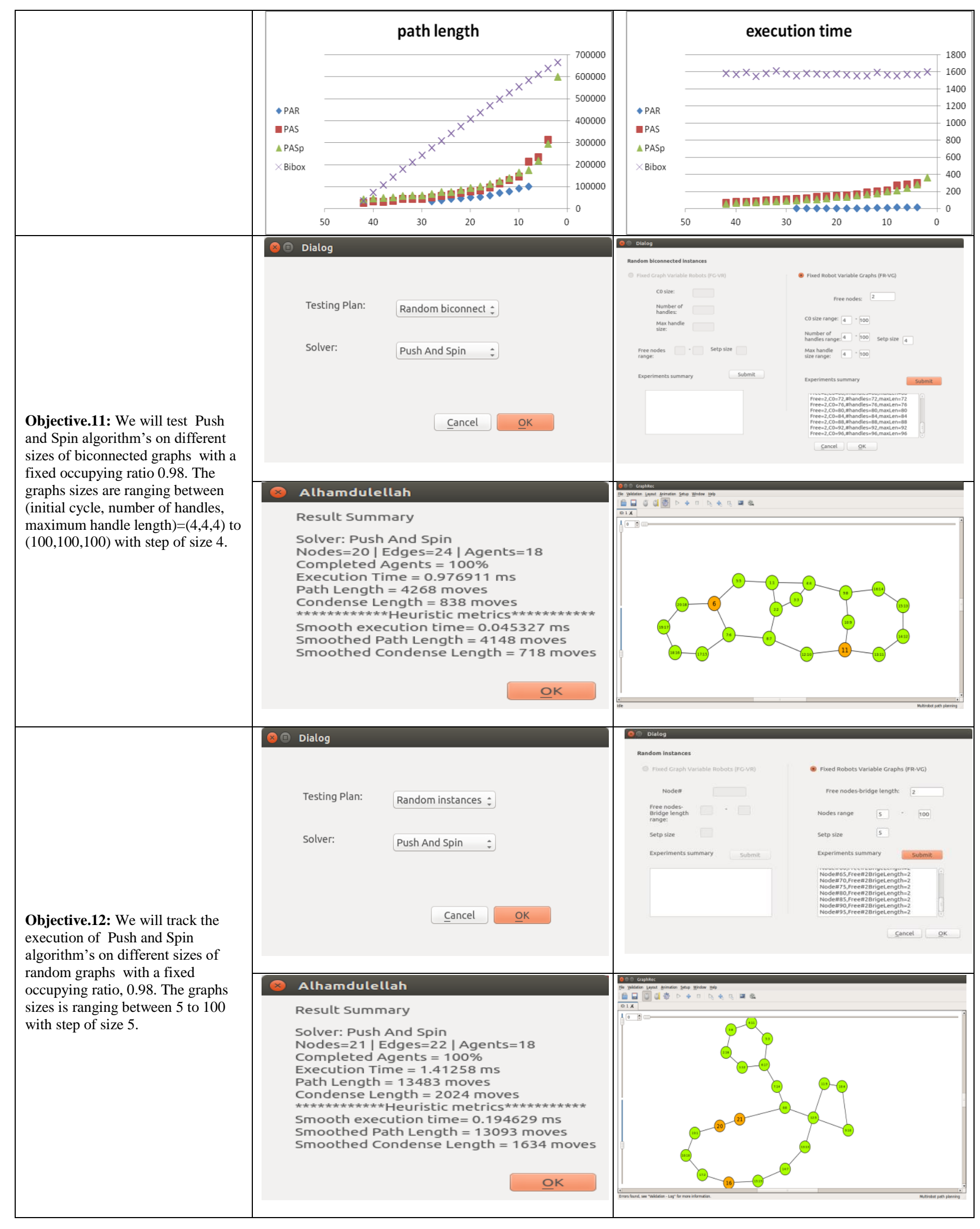




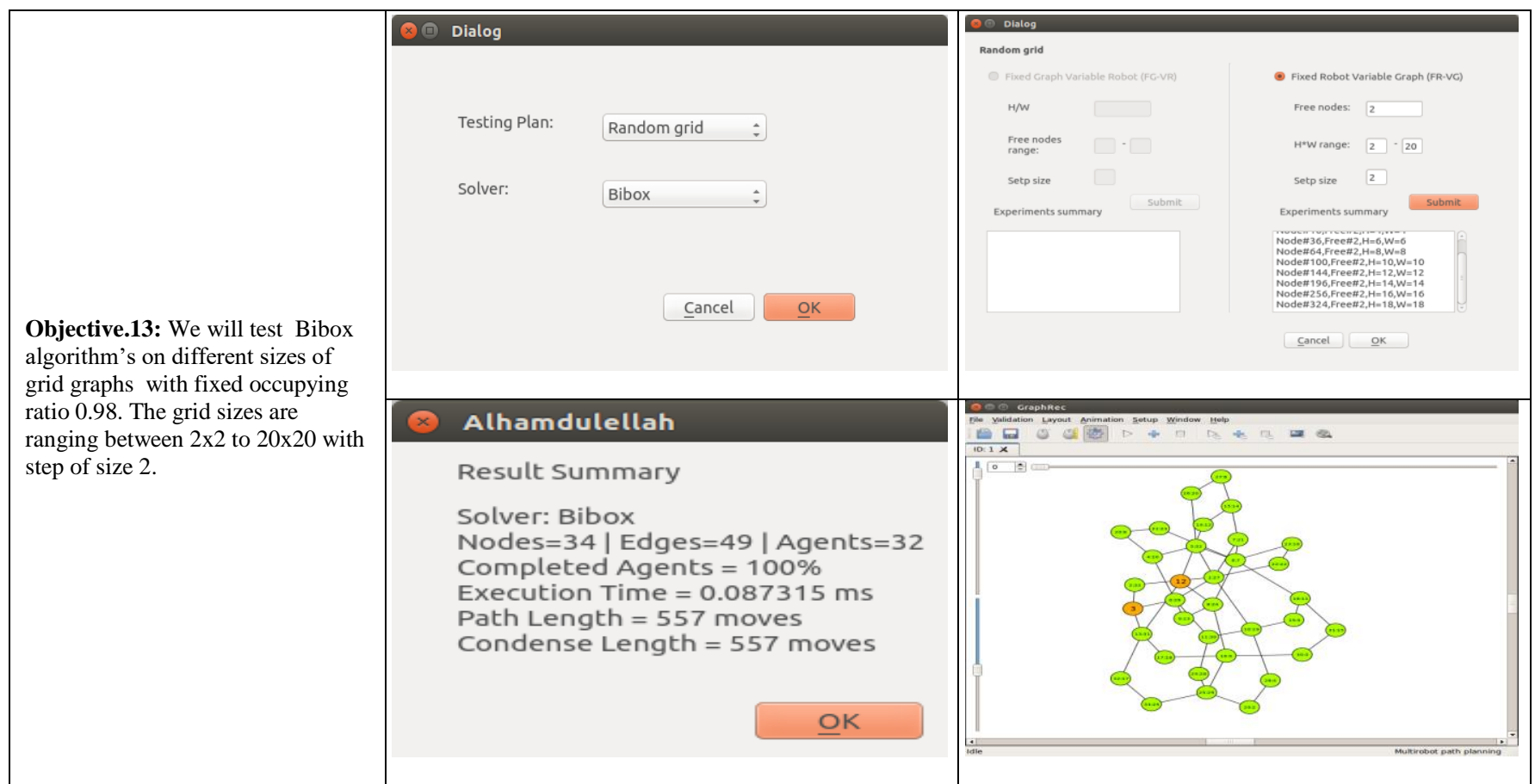

\section{CONCLUSION AND FUTURE WORKS}

As can be observed, the MRPP simulator provides complete simulation/evaluation tool for offline multi-robot path planning. Its major strength is its ability to provide the algorithms imposed graph representation for its environment. MRPP allows the researchers to;

- Test and track the algorithm's behavior for specific cases in fully controlled instances.

- Track the algorithm's behavior and compare its performance with the algorithms that already evaluated on predefined small critical instances in [7].

- Test the algorithm behavior on very large graphs when the occupying ratio (robots number/vertices count) increases.

- Compare the algorithm performance to the algorithms already tested in public large problems [1] such as [79].

- Compare the algorithm's performance to the performance of algorithms solves biconnected graphs [10], [7, 8].

- Test the algorithm behavior on fixed biconnected graph's size when the occupying ratio increases.

- Evaluate the algorithm performance against the algorithms solves random graphs.

- Track the algorithm behavior on fixed random graph's size when the occupying ratio increases.

- Compare the algorithm's performance to the performance of algorithms solves grid graphs $[7,8$, $10]$.
- Test the algorithm behavior on fixed grid graph's size when the occupying ratio increases.

- Evaluate the algorithm performance on biconnected graphs of fixed occupying ratio when the graph's size increases.

- Evaluate the algorithm performance on random graphs of fixed occupying ratio when the graph's size increases.

- Evaluate the algorithm performance on grid graphs of fixed occupying ratio when the graph's size increases.

- Plug new simulation model, MRPP algorithm implementation or performance measurement calculation.

Future work may include designing secure database, reporting set of experiments using different types of diagrams and adding new evaluation algorithms.

\section{REFERENCES}

[1] N. R. Sturtevant, "Benchmarks for grid-based pathfinding," Computational Intelligence and AI in Games, IEEE Transactions on, vol. 4, pp. 144-148, 2012.

[2] E. Guizzo, "Three Engineers, Hundreds of Robots, One Warehouse," IEEE Spectr., vol. 45, pp. 26-34, 2008.

[3] K. Dresner and P. Stone, "A multiagent approach to autonomous intersection management," Journal of Artificial Intelligence Research, pp. 591-656, 2008.

[4] J. Leitner, "Multi-robot cooperation in space: A survey," in Advanced Technologies for Enhanced Quality of Life, 2009. AT-EQUAL'09. 2009, pp. 144-151.

[5] J. M. Roberts, E. S. Duff, and P. I. Corke, "Reactive navigation and opportunistic localization for autonomous underground mining vehicles," Information Sciences, vol. 145, pp. 127-146, 2002.

[6] D. Nieuwenhuisen, A. Kamphuis, and M. H. Overmars, "High quality navigation in computer games," Science of Computer Programming, vol. 67, pp. 91-104, 2007. 
[7] R. Luna and K. E. Bekris, "Push and swap: Fast cooperative pathfinding with completeness guarantees," in IJCAI, 2011, pp. 294-300.

[8] B. d. Wilde, A. W. ter Mors, and C. Witteveen, "Push and Rotate: a Complete Multi-agent Pathfinding Algorithm," Journal of Artificial Intelligence Research, pp. 443-492, 2014.

[9] K.-H. C. Wang and A. Botea, "Mapp: a scalable multi-agent path planning algorithm with tractability and completeness guarantees," Journal of Artificial Intelligence Research, pp. 55-90, 2011.

[10] P. Surynek, "A novel approach to path planning for multiple robots in bi-connected graphs," in Robotics and Automation, 2009. ICRA'09. IEEE International Conference on, 2009, pp. 3613-3619.

[11] E. T. S. Alotaibi and H. Al-Rawi, "Multi-Robot Path-Planning Problem for a Heavy Traffic Control Application: A Survey International Journal of Advanced Computer Science and Applications(IJACSA), ," International Journal of Advanced Computer Science and Applications(IJACSA), vol. 7, p. 10, 1,7,2016 2016.

[12] S. Karaman and E. Frazzoli, "Sampling-based algorithms for optimal motion planning," The International Journal of Robotics Research, vol. 30, pp. 846-894, 2011.

[13] D. Kornhauser, G. Miller, and P. Spirakis, Coordinating pebble motion on graphs, the diameter of permutation groups, and applications: IEEE, 1984.

[14] P. Koupý, "Visualization of problems of motion on a graph," BSc, Department of Theoretical Computer Science and Mathematical Logic, Charles University in Prague, Faculty of Mathematics and Physics, Prague, 2010. 